\title{
Utility and limitations of glypican-3 expression for the diagnosis of hepatocellular carcinoma at both ends of the differentiation spectrum
}

\author{
Nafis Shafizadeh ${ }^{1}$, Linda D Ferrell ${ }^{1}$ and Sanjay Kakar ${ }^{1,2}$ \\ ${ }^{1}$ Department of Anatomic Pathology, University of California, San Francisco, CA, USA and ${ }^{2}$ Department of \\ Anatomic Pathology, Veteran Affairs Medical Center, San Francisco, CA, USA
}

\begin{abstract}
Glypican-3 is a heparin sulfate proteoglycan normally expressed in fetal liver and placenta, but not in normal adult liver. Preliminary studies have shown that glypican-3 can be useful for the diagnosis of hepatocellular carcinoma. We performed immunohistochemistry for glypican-3 on 80 resection cases of hepatocellular lesions to examine the utility of glypican-3 immunohistochemistry in hepatocellular carcinoma at two ends of the differentiation spectrum. Staining was compared to Hep Par 1 in poorly differentiated cases. Glypican-3 was expressed in $46(79 \%)$ hepatocellular carcinomas $(56,83$ and $89 \%$ of well, moderately and poorly differentiated respectively) and seven (64\%) fibrolamellar carcinomas. Of the 16 well differentiated cases, 10 closely resembled adenoma and were diagnosed due to focal abnormalities and/or loss of reticulin. Glypican-3 expression was seen in $\mathbf{5 0 \%}$ in this group. Hepatocellular carcinomas arising in cirrhotic liver were more likely to be glypican-3 positive (91 vs $57 \%, P=0.004)$. All hepatic adenomas and macroregenerative nodules were negative, and three $(43 \%)$ high grade dysplastic nodules were positive. Focal staining was seen in regenerative nodules in four $(11 \%)$ cirrhosis cases. Glypican-3 was significantly more sensitive than Hep Par 1 for diagnosis of poorly differentiated hepatocellular carcinomas ( 89 vs $63 \%, P=0.02)$. The difference was more significant when only cases with diffuse positive staining were considered $(83$ vs $21 \%, P<0.001)$. In conclusion, glypican-3 has high sensitivity for the diagnosis of hepatocellular carcinoma, but is less sensitive in the extremely well differentiated hepatocellular carcinoma and fibrolamellar variant of hepatocellular carcinoma. Caution should be exercised in using glypican-3 in biopsy specimens as cirrhotic nodules can show strong expression. Glypican-3 can be especially useful in the identification of poorly differentiated hepatocellular carcinoma as it has higher sensitivity compared to Hep Par 1.
\end{abstract}

Modern Pathology (2008) 21, 1011-1018; doi:10.1038/modpathol.2008.85; published online 6 June 2008

Keywords: glypican-3; hepatocellular carcinoma; Hep Par 1

The diagnosis of hepatocellular carcinoma often requires the use of immunohistochemistry, especially in small biopsy specimens. Several immunohistochemical markers have been used for the diagnosis of hepatocellular differentiation. In the last few years, Hep Par 1 has emerged as a sensitive and relatively specific marker of hepatocellular differentiation. ${ }^{1-4}$ Polyclonal carcinoembryonic antigen (CEA) yields a characteristic canalicular pattern of staining that is considered specific for hepatocellular differentiation. ${ }^{5,6}$ MOC31 has emerged as a useful marker as it is expressed in

Correspondence: Dr S Kakar, MD, Department of Anatomic Pathology, UCSF and VA Medical Centers, 4150 Clement Street, San Francisco, CA 94121, USA.

E-mail: sanjay.kakar@ucsf.edu

Received 30 November 2007; revised 10 March 2008; accepted 19 March 2008; published online 6 June 2008 virtually all adenocarcinomas and is negative or weakly positive in hepatocellular carcinoma. ${ }^{5,7}$ Other markers of hepatocellular differentiation that have been used include $\alpha$-fetoprotein, thyroid transcription factor-1 and CD10. ${ }^{5,6,8}$

The presently employed immunohistochemical panels have greatly facilitated the diagnosis of HCC on limited samples. However, there are several situations where these markers are of limited use. Hep Par 1 and polyclonal CEA have low sensitivity $(\sim 50 \%)$ for the diagnosis of poorly differentiated hepatocellular carcinoma. ${ }^{3,4}$ Hence, they may not be helpful in the setting of a poorly differentiated hepatic neoplasm in distinguishing hepatocellular carcinoma and metastatic adenocarcinoma. At the other end of the spectrum, it can be difficult to distinguish hepatic adenomas from well differentiated hepatocellular carcinoma arising in noncirrhotic liver. A similar dilemma can arise in the 
setting of a cirrhotic liver, where it can be challenging to separate high-grade dysplastic nodule from a well-differentiated hepatocellular carcinoma. Most markers of hepatocellular differentiation like Hep Par 1 and polyclonal CEA do not distinguish between benign and malignant hepatocellular lesions. AFP is expressed only in HCC, but its sensitivity is low $(20-30 \%)$, limiting its diagnostic potential. ${ }^{3,6}$

Glypican-3 (GPC-3) is a membrane anchored heparin sulfate proteoglycan normally expressed in fetal liver and placenta, but not in normal adult liver. $^{9-11}$ It is an oncofetal antigen that is a reliable serum and histochemical marker for hepatocellular carcinoma. ${ }^{11-13}$ Preliminary studies have demonstrated the promise of GPC-3 immunohistochemistry for the diagnosis of hepatocellular carcinoma. GPC-3 expression has been reported in $75-100 \%$ of hepatocellular carcinomas. ${ }^{10,11,14-17}$ Expression of GPC-3 has not been observed in benign hepatocellular lesions by in situ hybridization or immunohistochemistry. ${ }^{7,18}$ It has also been suggested that poorly differentiated hepatocellular carcinomas are more likely to express GPC- $3 .{ }^{16}$ High grade dysplastic nodules typically express GPC-3 in a weak and focal fashion, although the results have not been consistent across different series. ${ }^{15,16}$

This study evaluates the diagnostic role of GPC-3 immunohistochemistry in HCC with emphasis on diagnosis of the poorly differentiated hepatocellular carcinoma and the distinction between well-differentiated hepatocellular carcinoma and hepatic adenomas. GPC-3 expression in fibrolamellar variant of hepatocellular carcinoma and nodular lesions in cirrhotic liver was also evaluated.

\section{Materials and methods}

\section{Cases}

The study population comprised of 90 formalinfixed, paraffin-embedded resection cases of hepatocellular lesions obtained from the files of UCSF and VA Medical Centers. The slides were reviewed to confirm the diagnosis as per the guidelines of the International Working Party guidelines ${ }^{19,20}$ and the following groups were studied.

(a) Macroregenerative nodules $(n=10)$.

(b) High grade dysplastic nodules $(n=7)$.

(c) Hepatic adenomas $(n=8)$.

(d) Hepatocellular carcinoma including well $(n=16)$, moderately $(n=14)$ and poorly differentiated $(n=24)$ cases.

(e) Fibrolamellar hepatocellular carcinoma $(n=11)$.

\section{Immunohistochemistry}

GPC-3 staining was performed in all 90 hepatocellular lesions using monoclonal antibody directed against GPC-3 (Clone $1 \mathrm{G} 12$ used at $5 \mu \mathrm{g} / \mathrm{ml}$, BioMosaics,
Burlington, VT, USA). Immunohistochemistry for Hep Par 1 (Clone OCH1E5, Dako, Carpinteria, CA, USA) was performed in all 24 poorly differentiated HCC. Briefly, tissue sections were deparaffinized, rehydrated, and antigen retrieval was performed using heat-induced epitope retrieval in a $10 \mathrm{mmol} / \mathrm{l}$ citrate buffer, $\mathrm{pH}$ 6.0. Following peroxidase block and incubation with primary antibody for $30 \mathrm{~min}$, the sections were incubated with horse-radish peroxidase-labeled secondary antibody (30 and 15 min for glypican-3 and Hep Par 1, respectively), substrate chromagen for $10 \mathrm{~min}$, and counterstained with hematoxylin.

The results of immunohistochemical staining with GPC-3 in hepatocellular lesions were recorded as negative ( $0-5 \%$ of tumor cells), focal positive (6-50\% of tumor cells) and diffuse positive $(>50 \%$ of tumor cells) based on visual estimation of the entire tumor on the slide. The results with Hep Par 1 were similarly noted in poorly differentiated hepatocellular carcinomas and compared with GPC-3 staining. $\chi^{2}$ test was used to compare the GPC-3 and Hep Par 1 results in poorly differentiated hepatocellular carcinoma. $P$-values of $<0.05$ were regarded as statistically significant.

\section{Results}

\section{GPC-3 Staining in Benign and Malignant Hepatocellular Lesions}

\section{Conventional hepatocellular carcinoma}

Of the 58 patients (36 men, 18 women, four unknown gender; age range, 33-83 years), cirrhosis was present in $35(60 \%)$ cases, while status of cirrhosis was unknown in two cases. The cirrhosis was related to hepatitis C $(n=19)$, hepatitis $\mathrm{B}$ $(n=2)$, both hepatitis $\mathrm{B}$ and $\mathrm{C}(n=1)$, alcohol $(n=2)$ and non-alcoholic steatohepatitis $(n=1)$, whereas the etiology was unknown in 10 cases. GPC-3 expression was observed in 46 (79\%) hepatocellular carcinoma cases (Table 1, Figure 1). All cases showed granular cytoplasmic pattern of staining, with additional membranous accentuation in five cases. The staining was diffuse in $37(80 \%)$ and focal in nine $(20 \%)$ tumors. GPC-3 expression was more often observed in hepatocellular carcinoma arising in cirrhosis (91 vs 57\%, $P=0.004$ ).

The grading of hepatocellular carcinomas was performed using WHO criteria. ${ }^{21}$ GPC-3 staining was seen in 9/16 (56\%), 15/18 (83\%) and 22/24 (89\%) well, moderately and poorly differentiated hepatocellular carcinomas respectively $(P=0.02)$. Among the 16 well-differentiated tumors, six cases were easily diagnosed on routine H\&E sections, while 10 cases closely resembled hepatic adenoma, and the diagnosis of hepatocellular carcinoma was based on focal atypical features like small cell change, widened cell plates, stromal invasion and/or loss of reticulin. All these cases occurred in noncirrhotic liver and were referred to as extremely 
Table 1 GPC-3 staining results in benign and malignant hepatocellular lesions

\begin{tabular}{lcr}
\hline \multirow{2}{*}{ Liver lesions } & \multicolumn{2}{c}{ GPC-3 staining } \\
\cline { 2 - 3 } & $\begin{array}{c}\text { Total } \\
\text { positive }\end{array}$ & $\begin{array}{c}\text { Diffuse } \\
\text { positive }\end{array}$ \\
\hline $\begin{array}{l}\text { Benign hepatocellular lesions } \\
\text { Cirrhotic nodules }(n=35)\end{array}$ & $4(11)$ & $0(0)$ \\
$\begin{array}{l}\text { Macroregenerative nodule }(n=10) \\
\text { Hepatic adenoma }(n=8)\end{array}$ & $0(0)$ & $0(0)$ \\
& $0(0)$ & $0(0)$ \\
$\begin{array}{l}\text { Borderline and malignant lesions } \\
\text { High-grade dysplastic nodule } \\
(n=7)\end{array}$ & $3(43)$ & $0(0)$ \\
$\begin{array}{l}\text { Extremely well-differentiated HCC } \\
(n=10)\end{array}$ & $5(50)$ & $2(20)$ \\
$\begin{array}{l}\text { HCC, well differentiated }(n=16)^{\mathrm{b}} \\
\text { HCC, moderately differentiated } \\
(n=18)\end{array}$ & $9(56)$ & $5(31)$ \\
$\begin{array}{l}\text { HCC, poorly differentiated }(n=24) \\
\text { Fibrolamellar HCC }(n=11)\end{array}$ & $22(89)$ & $10(56)$ \\
\hline
\end{tabular}

HCC, hepatocellular carcinoma.

${ }^{a}$ Figures in parenthesis reflect percentages.

${ }^{b}$ Includes extremely well-differentiated HCC.

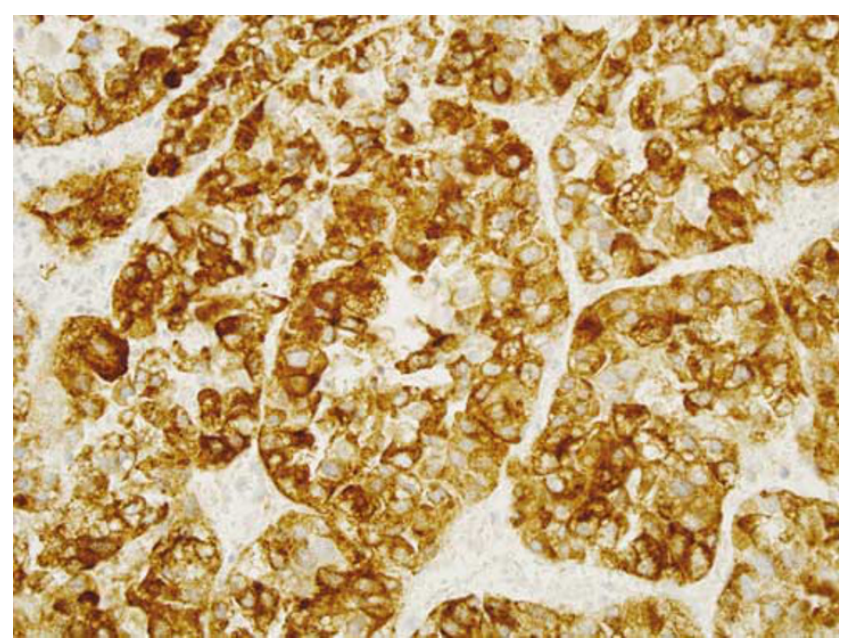

Figure 1 Diffuse cytoplasmic expression of glypican-3 in hepatocellular carcinoma $(\times 400)$.

well differentiated hepatocellular carcinoma. There was one case of each of recurrence and metastasis in these cases. GPC-3 expression was seen in five $(50 \%)$ cases in this group (Figures 2 and 3 ). When these cases were excluded from comparison, there was no difference in GPC-3 staining and differentiation $(P=0.2)$. Among the other six well-differentiated cases, there were two cases of early hepatocellular carcinoma arising in cirrhotic liver. Both these cases formed $1.5 \mathrm{~cm}$ nodules with indistinct margins and the diagnosis was based on stromal invasion at the edge of the nodule. ${ }^{22,23}$ Both these cases showed diffuse GPC-3 expression (Figure 4).
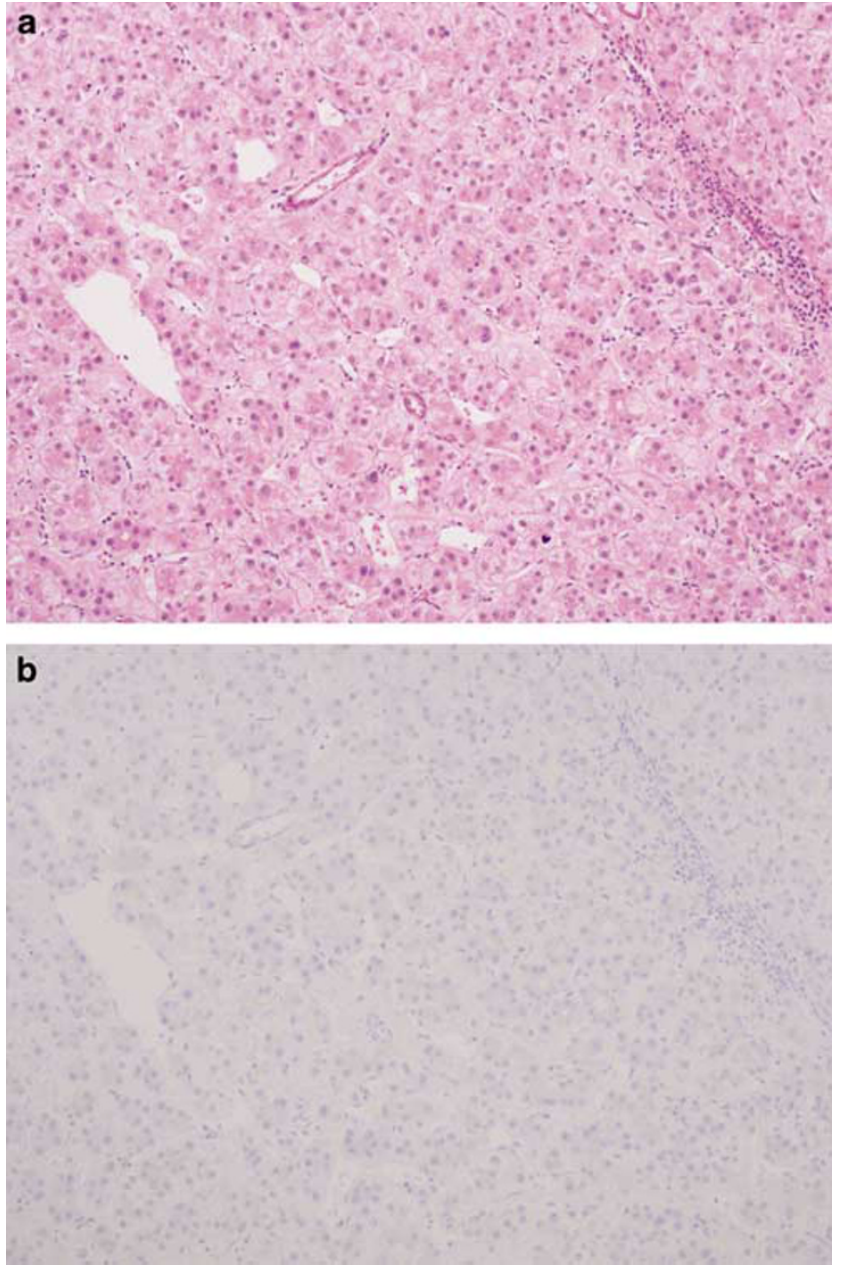

Figure 2 Extremely well differentiated hepatocellular carcinoma $(\mathbf{a}, \mathrm{H} \& \mathrm{E} \times 100)$ showing lack of glypican-3 expression $(\mathbf{b}, \times 100)$.

\section{Fibrolamellar hepatocellular carcinoma}

All 11 cases (six male, five female, age range, 17-47 years) occurred in non-cirrhotic liver. GPC-3 expression was present in seven (64\%) cases, of which four were diffuse positive (Figure 5).

\section{Hepatic adenoma}

All eight cases (two males, six females, age range, 27-51 years) occurred in non-cirrhotic liver. History of oral contraceptive and anabolic steroids use was present in three women and two men respectively. One case showed multiple adenomas (hepatic adenomatosis). All cases were negative for GPC-3 (Figure 6).

\section{Hepatocellular nodular lesions}

All macroregenerative and high grade dysplastic nodules occurred in the setting of cirrhosis. All 10 cases of macroregenerative nodule were negative for GPC-3. Of the seven high-grade dysplastic nodules, three $(43 \%)$ showed GPC-3 expression, with focal staining in all cases. The regenerative cirrhotic nodules were examined for GPC-3 expression in 35 
1014
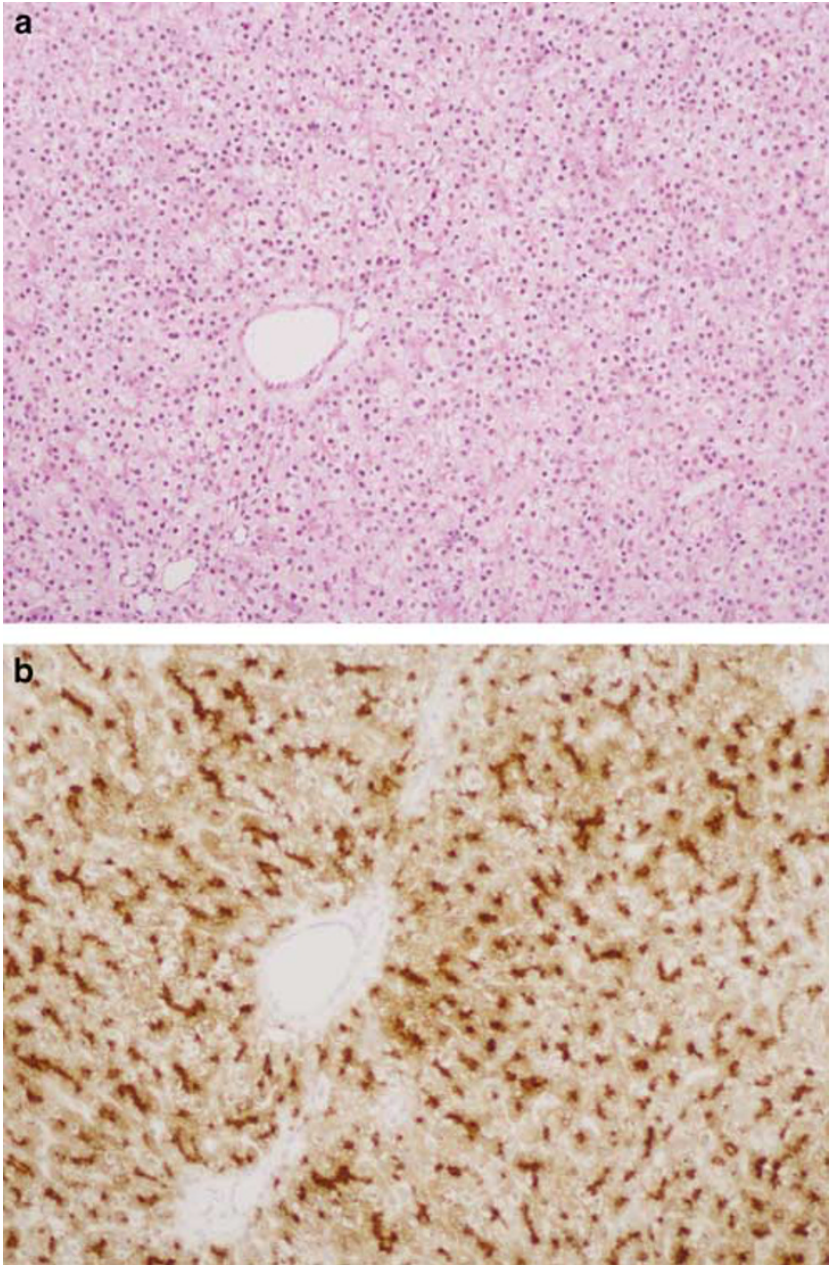

Figure 3 Extremely well differentiated hepatocellular carcinoma $(\mathbf{a}, \mathrm{H} \& \mathrm{E} \times 100)$ showing glypican-3 expression $(\mathbf{b}, \times 100)$.

cases. Staining was absent in the overwhelming majority of the regenerative nodules; however, focal but strong GPC-3 expression was observed in 1-2 nodules in four (11\%) cases (Figure 7 ).

\section{Hep Par 1 and GPC-3 Staining in Poorly Differentiated Hepatocellular Carcinoma}

Of the 24 poorly differentiated hepatocellular carcinomas, GPC-3 expression was observed in 22 (89\%) cases compared to $15(63 \%)$ with Hep Par 1 $(P=0.02)$ (Table 2, Figure 8). The difference was more pronounced if cases with diffuse positive staining were considered: $20(83 \%)$ with GPC-3 vs $5(21 \%)$ with Hep Par $1(P<0.001)$. All nine cases that were Hep Par 1 negative, expressed GPC-3, while both GPC-3 negative cases were positive for Hep Par 1.

\section{Discussion}

The glypican proteins are members of the cellsurface heparin sulfate proteoglycans, which have
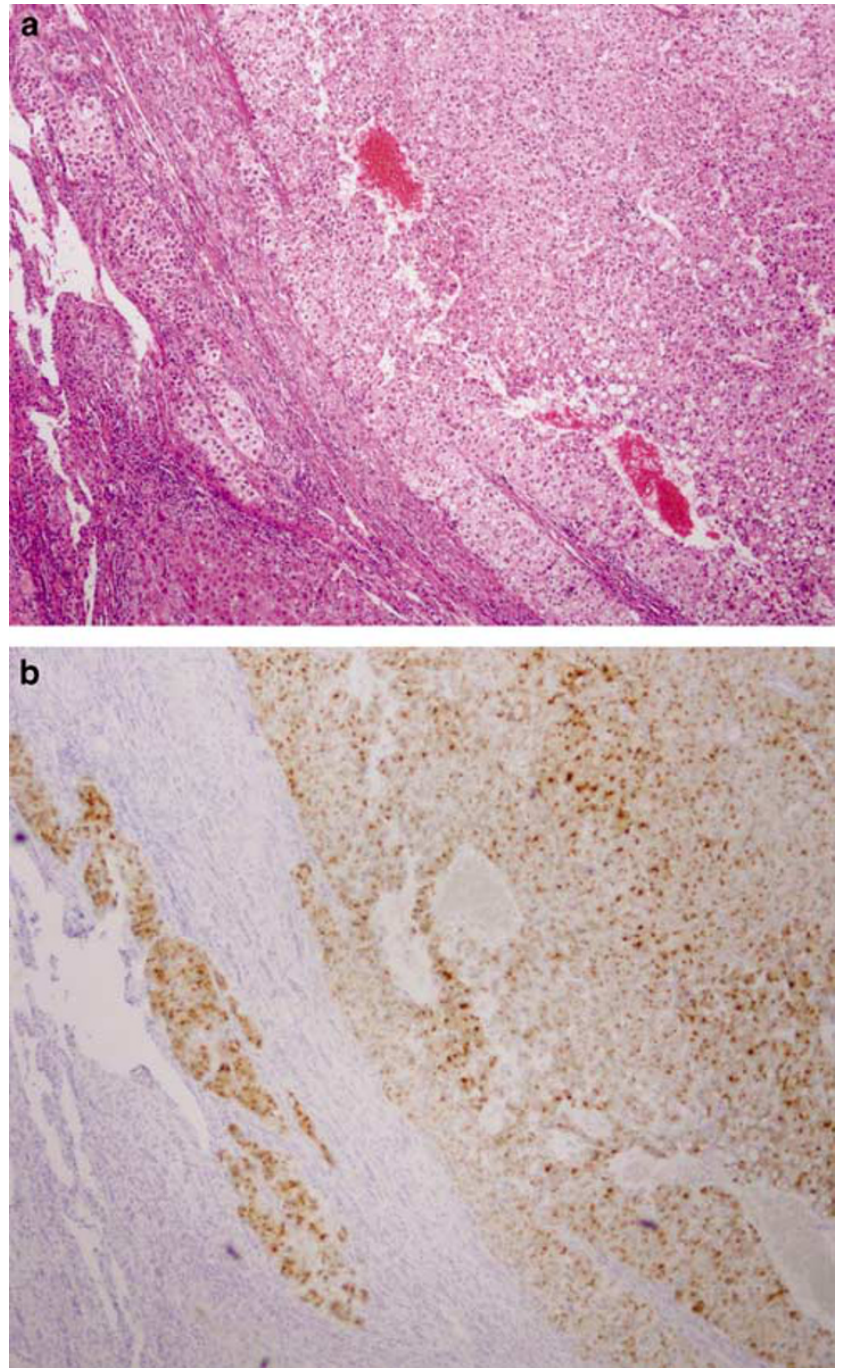

Figure 4 Early well differentiated hepatocellular carcinoma with stromal and parenchymal invasion in the left portion (a, H\&E $\times 40$ ). Glypican-3 expression is present in the nodule as well as in the invasive portion $(\mathbf{b}, \times 40)$.

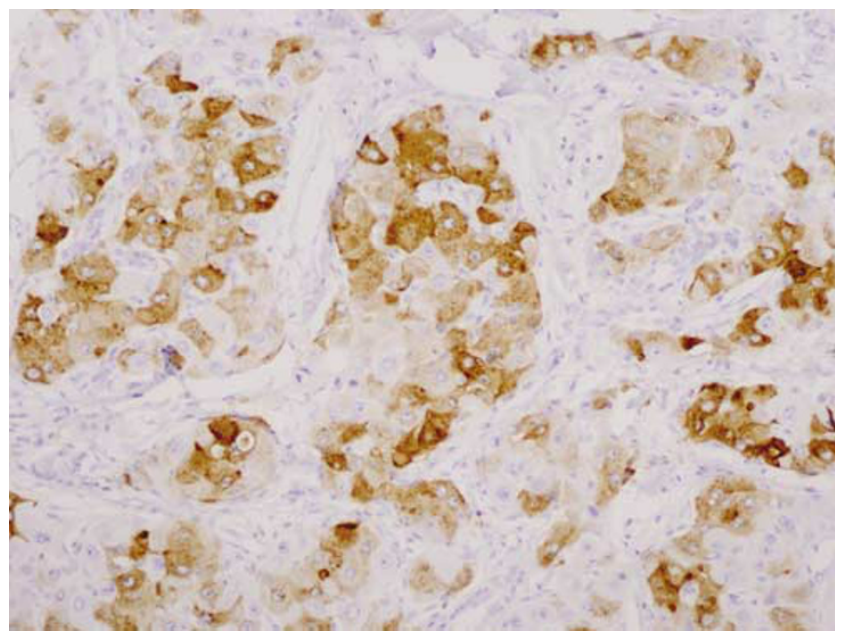

Figure 5 Diffuse cytoplasmic expression of glypican-3 in fibrolamellar hepatocellular carcinoma $(\times 200)$. 


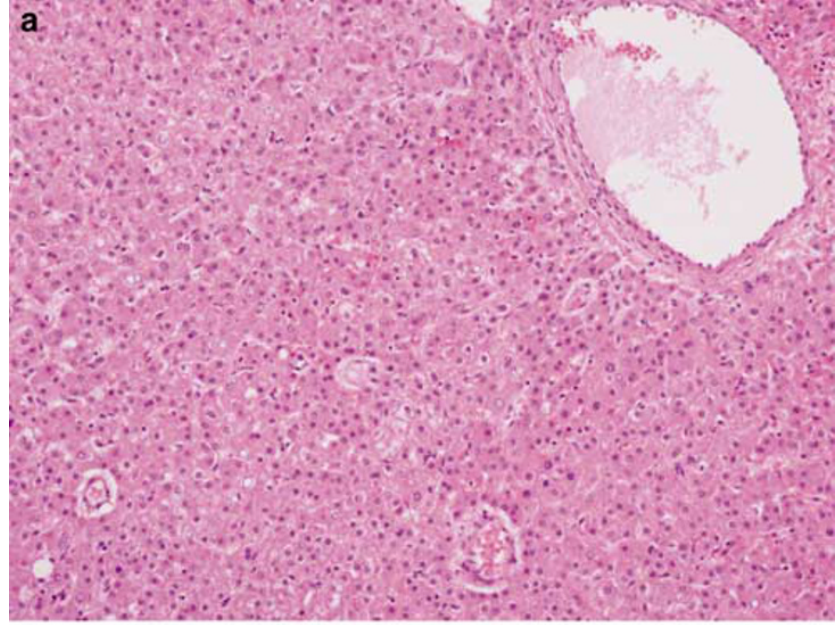

b

Figure 6 Hepatic adenoma $(\mathbf{a}, \mathrm{H} \& \mathrm{E} \times 100)$ showing lack of glypican-3 expression $(\mathbf{b}, \times 100)$.

been shown to interact with growth factors and modulate growth factor activity. ${ }^{18,24}$ GPC-3 has been demonstrated to be upregulated in hepatocellular carcinoma by northern and western blot analysis. $^{9,18,24}$ It modulates cell-cycle progression and promotes cellular migration and invasiveness in hepatocellular carcinoma cell lines. ${ }^{25-27}$ The $1 \mathrm{G} 12$ monoclonal antibody against GPC-3 was developed in mice and is suitable for use in formalin-fixed paraffin-embedded tissue.

Several studies have demonstrated the efficacy of GPC-3 as a diagnostic tool in hepatocellular carcinoma. The reported sensitivity ranges from 75$100 \%$, with figures of $75-85 \%$ in larger series. ${ }^{10,11,14-17}$ Our results are similar to the literature, with $79 \%$ of conventional hepatocellular carcinoma being positive for GPC-3. Tumors arising in cirrhotic liver are more likely to express GPC-3 (91 vs 57\%, $P=0.004$ ), which is consistent with previous studies. ${ }^{15}$ There has been little emphasis in the literature on the utility of GPC-3 at diagnostically challenging ends of the spectrum, that is, extremely well-differentiated hepatocellular carcinoma and
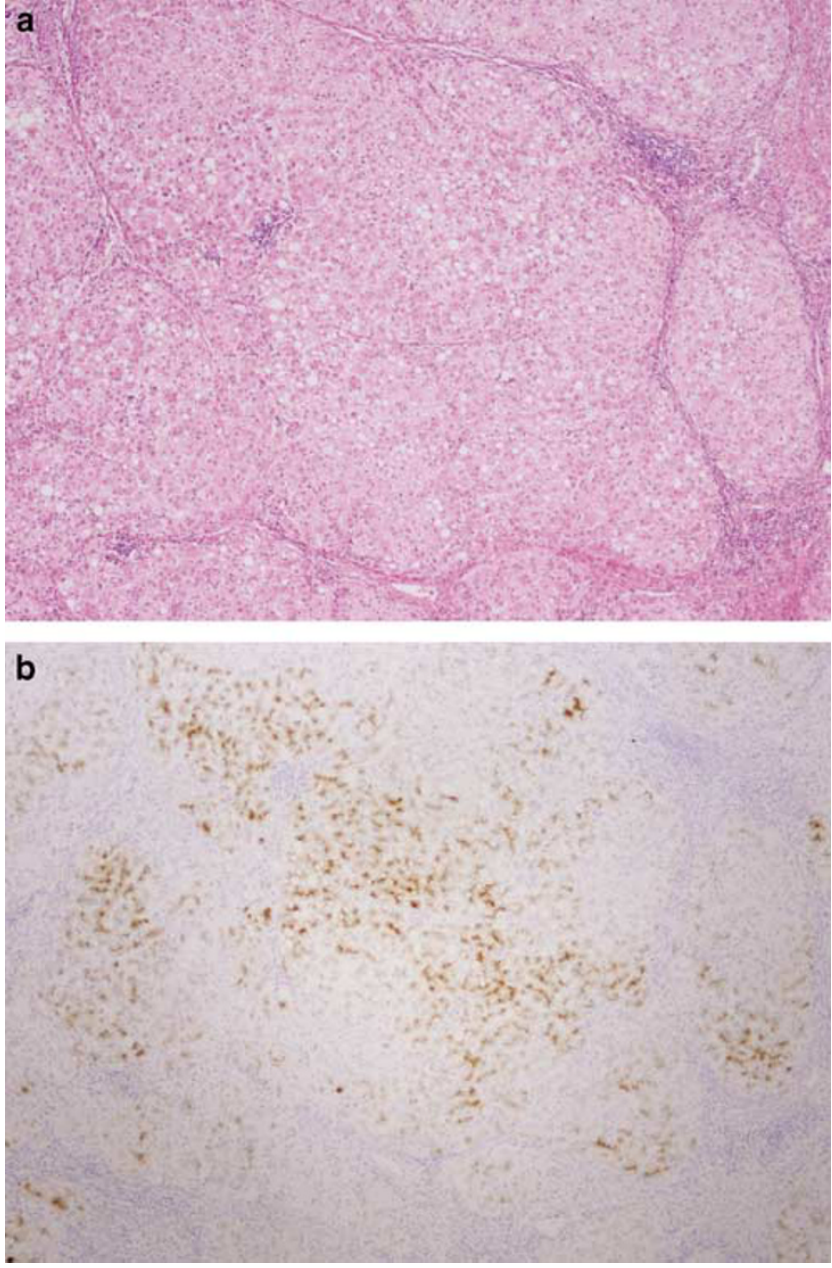

Figure 7 Regenerative cirrhotic nodules $(a, H \& E \times 40)$ showing patchy expression of glypican-3 $(\mathbf{b}, \times 40)$.

Table 2 Comparison of Hep Par 1 and GPC-3 staining results in 24 cases of poorly differentiated hepatocellular carcinoma

\begin{tabular}{lcrc}
\hline & GPC-3 & Hep Par 1 & P-value \\
\hline $\begin{array}{l}\text { Diffuse positive } \\
\begin{array}{l}\text { Total positive } \\
\text { (focal and diffuse) }\end{array}\end{array}$ & $22(83)$ & $5(21)$ & $<0.001$ \\
& & $15(63)$ & 0.02 \\
\hline
\end{tabular}

Figures in parenthesis reflect percentages.

poorly differentiated hepatocellular carcinoma. Similar to earlier studies, we did not find any significant difference in sensitivity of GPC-3 and differentiation of hepatocellular carcinoma when typical cases of well, moderately and poorly differentiated hepatocellular carcinomas were considered. ${ }^{14,17}$ However, the sensitivity of GPC-3 was lower in the well-differentiated category when extremely well-differentiated variant of hepatocellular carcinoma was included in the analysis. These tumors occurred in non-cirrhotic liver and were morphologically very similar to hepatic adenomas, 
1016
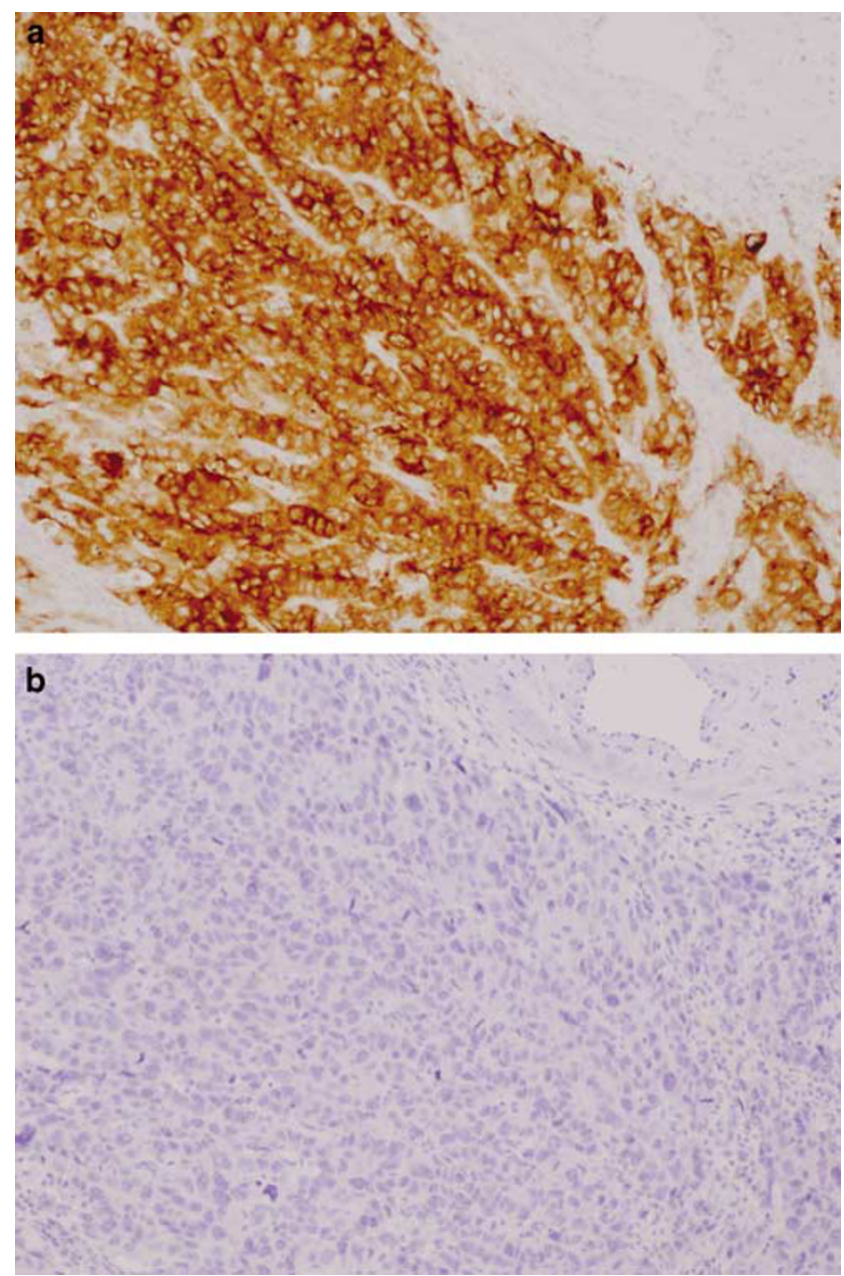

Figure 8 Poorly differentiated hepatocellular carcinoma with diffuse glypican-3 expression $(\mathbf{a}, \times 200)$. Hep Par 1 expression in the same tumor is negative $(\mathbf{b}, \times 200)$.

but exhibited focal architectural abnormalities and abnormal reticulin to enable the diagnosis of hepatocellular carcinoma. These tumors can be difficult to separate from hepatic adenoma on morphological grounds and GPC-3 expression in this group has not been specifically addressed. The sensitivity of GPC-3 dropped to $50 \%$ in these cases. These cases are different from early hepatocellular carcinoma described in literature as they occur in non-cirrhotic liver and are generally larger than $2 \mathrm{~cm}$. In contrast, early hepatocellular carcinoma are generally $<1.5 \mathrm{~cm}$ and arise in cirrhotic liver. ${ }^{22,23}$ Both cases of early hepatocellular carcinoma in our series were GPC-3 positive. GPC-3 expression in 60$69 \%$ of early HCC has been reported. ${ }^{15,28}$ All hepatic adenomas in this study as well as the literature have been negative for GPC-3. Hence GPC-3 expression in a well-differentiated hepatic neoplasm in noncirrhotic liver strongly favors hepatocellular carcinoma. However, in view of the low sensitivity of GPC-3 in extremely well-differentiated hepatocellular carcinoma, a negative result does not exclude carcinoma.
In setting of a well-differentiated hepatocellular nodule in cirrhotic liver, high-grade dysplastic nodule can be difficult to separate from hepatocellular carcinoma. The results of GPC-3 staining in high-grade dysplastic nodules are inconsistent in the literature with two studies showing expression in a minority of cases (seven and $22 \%$ ), while $75 \%$ of cases were positive in another series. ${ }^{14-16}$ This variation is not surprising, given the difficulty in distinguishing high-grade dysplastic nodules and hepatocellular carcinoma. ${ }^{20}$ In our study, $43 \%$ of high-grade dysplastic nodules were GPC-3 positive. In all our cases and in the literature, GPC-3 expression was focal involving $<50 \%$ of tumor cells. Hence diffuse expression of GPC-3 in a resection specimen would favor hepatocellular carcinoma. However, GPC-3 is likely to be of limited use in this distinction on needle biopsies.

At the other end of the spectrum, poorly differentiated hepatocellular carcinomas pose a diagnostic problem as they can mimic metastatic neoplasms. The commonly used hepatocellular markers like Hep Par 1 and polyclonal CEA have low sensitivity for poorly differentiated hepatocellular carcinoma, ${ }^{3,4,6}$ and their staining efficacy has not been systematically compared with GPC-3. Our study shows that GPC-3 has higher sensitivity than Hep Par 1 for the diagnosis of poorly differentiated hepatocellular carcinoma (89 vs $63 \%, P=0.02$ ). The difference is more pronounced when cases with diffuse positive staining are considered (79 vs $26 \%$, $P<0.001$ ). Yamauchi et $a l^{14}$ reported $100 \%$ staining of poorly differentiated hepatocellular carcinoma with GPC-3 compared to $67 \%$ with Hep Par 1, but only six cases were studied. Higher sensitivity and more cases with diffuse staining indicate that GPC-3 will have greater utility in the diagnosis of hepatocellular carcinoma in liver biopsies, and may be preferred over Hep Par 1 if limited tissue is available. All 24 cases of poorly differentiated hepatocellular carcinoma showed expression of either GPC-3 or Hep Par1. Thus, the combined use of Hep Par1 and GPC-3 is complementary for the diagnosis of poorly differentiated hepatocellular carcinoma with a sensitivity of $100 \%$ in our study.

Immunoreactivity of GPC-3 in variants of hepatocellular carcinoma has not been systematically examined. The fibrolamellar variant of hepatocellular carcinoma typically occurs in non-cirrhotic liver in young patients. Elevation of oncofetal antigen $\alpha$-fetoprotein is infrequent in fibrolamellar hepatocellular carcinoma and immunohistochemistry is generally negative. ${ }^{29,30}$ Our results show that unlike $\alpha$-fetoprotein, GPC-3, which is also an oncofetal antigen, is expressed in two-thirds of fibrolamellar carcinomas. In a smaller series of five fibrolamellar hepatocellular carcinomas, GPC-3 expression was noted in one tumor. ${ }^{15}$ Our study group included two cases of scirrhous hepatocellular carcinoma both of which showed diffuse-positive staining with GPC-3. Hep Par 1 has low sensitivity for the diagnosis of 
scirrhous hepatocellular carcinoma. ${ }^{3,31}$ GPC-3 may be a better marker of scirrhous hepatocellular carcinoma, but additional cases need to be studied to confirm this preliminary observation.

Although the results with GPC-3 are promising, its limitations should be kept in mind while employing it for diagnostic use. The experience with this antibody is still limited and its expression in adenocarcinomas of various sites and other tumors that can mimic hepatocellular carcinoma such as renal cell carcinoma have not been widely studied. Small series have reported negative results in cholangiocarcinomas and majority of metastatic adenocarcinomas, ${ }^{14,17}$ but GPC-3 expression has been described in melanomas, ovarian carcinoma and rarely in metastatic colonic adenocarcinoma. ${ }^{14,32-34}$ Scattered and weak GPC-3 expression can be seen in cirrhotic nodules and can rarely be intense as seen in four cases in this study. This observation has not been emphasized in the literature, although strong staining has also been observed in chronic hepatitis $\mathrm{C}$ with active necroinflammatory changes. ${ }^{35}$ Although the expression in inflamed parenchyma and cirrhotic nodules tend to be focal, it can lead to errors in interpretation of biopsy specimens.

In conclusion, this study confirms that glypican-3 immunohistochemistry has high overall sensitivity for the diagnosis of hepatocellular carcinoma, but is less efficacious in the identification of extremely well-differentiated hepatocellular carcinoma and fibrolamellar hepatocellular carcinoma. An Occasional strong expression can be seen in cirrhotic nodules and it can be mistaken for hepatocellular carcinoma in needle biopsies. Glypican-3 is superior to Hep Par 1 for the identification of poorly differentiated hepatocellular carcinoma. The combined use of glypican-3 and Hep Par 1 yields high sensitivity and identified all poorly differentiated hepatocellular carcinomas in this series. Further studies in a wide variety of tumors are necessary before the specificity of GPC-3 for hepatocellular carcinoma can be firmly established.

\section{Disclosure/conflict of interest}

None.

\section{References}

1 Wennerberg AE, Nalesnik MA, Coleman WB. Hepatocyte paraffin 1: a monoclonal antibody that reacts with hepatocytes and can be used for the differential diagnosis of hepatic tumors. Am J Pathol 1993;143: 1050-1054.

2 Fan Z, van de Rijn M, Montgomery K, et al. Hep Par1 antibody stain for the differential diagnosis of hepatocellular carcinoma: 676 tumors testing using tissue microarrays and conventional tissue sections. Mod Pathol 2003;16:137-144.
3 Kakar S, Muir T, Murphy LM, et al. Immunoreactivity of Hep Par1 in hepatic and extrahepatic tumors and its correlation with albumin in situ hybridization in hepatocellular carcinoma. Am J Clin Pathol 2003;119: 361-366.

4 Lugli A, Tornillo L, Mirlacher M, et al. Hepatocye Paraffin 1 expression in human normal and neoplastic tissues: Tissue microarray analysis on 3940 tissue samples. Am J Clin Pathol 2004;122:721-727.

5 Morrison C, Marsh Jr W, Frankel WL. A comparison of CD10 to pCEA, MOC-31 and hepatocyte for the distinction of malignant tumors in the liver. Mod Pathol 2002;15:1279-1287.

6 Lau SK, Prakash S, Geller SA, et al. Comparative immunohistochemical profile of hepatocellular carcinoma, cholangiocarcinoma, and metastatic adenocarcinoma. Hum Pathol 2002;33:1175-1181.

7 Proca DM, Niemann TH, Porcell AI, et al. MOC31 immunoreactivity in primary and metastatic carcinoma of the liver. Report of findings and review of other utilized markers. Appl Immunohistochem Mol Morphol 2000;8:120-125.

8 Lei JY, Bourne PA, diSant'Agnese PA, et al. Cytoplasmic staining of TTF-1 in the differential diagnosis of hepatocellular carcinoma vs cholangiocarcinoma and metastatic carcinoma of the liver. Am J Clin Pathol 2006;125:519-525

9 Midorikawa Y, Ishikawa S, Iwanari H. Glypican-3, overexpressed in hepatocellular carcinoma, modulates FGF2 and BMP-7 signaling. Int J Cancer 2003;103: 455-465.

10 Capurro M, Wanless IR, Sherman M, et al. Glypican 3: a novel serum and histochemical marker for hepatocellular carcinoma. Gastroenterology 2003;125:89-97.

11 Nakatsura T, Yoshitake Y, Senju S, et al. Glypican-3, overexpressed specifically in human hepatocellular carcinoma, is a novel tumor marker. Biochem Biophys Res Commun 2003;306:16-25.

12 Nakatsura T, Nishimura Y. Usefulness of the novel oncofetal antigen glypican-3 for diagnosis of hepatocellular carcinoma and melanoma. BioDrugs 2005;19: 71-77.

13 Filmus J, Capurro M. Glypican-3 and alphafetoprotein as diagnostic tests for hepatocellular carcinoma. Mol Diagn 2004;8:207-212.

14 Yamauchi N, Watanabe A, Hishinuma $\mathrm{M}$, et al. The glypican 3 oncofetal protein is a promising diagnostic marker for hepatocellular carcinoma. Mod Pathol 2005;18:1591-1598.

15 Wang XY, Degos F, Dubois S, et al. Glypican-3 expression in hepatocellular tumors: diagnostic value for preneoplastic lesions and hepatocellular carcinomas. Hum Pathol 2006;37:1435-1441.

16 Libbrecht L, Severi T, Cassiman D, et al. Glypican-3 expression distinguishes small hepatocellular carcinomas from cirrhosis, dysplastic nodules, and focal nodular hyperplasia-like nodules. Am J Surg Pathol 2006;30:1405-1411.

17 Kandil D, Leiman G, Allegretta $\mathrm{M}$, et al. Glypican-3 immunocytochemistry in liver fine-needle aspirates: a novel stain to assist in the differentiation of benign and malignant liver lesions. Cancer 2007;111: 316-322.

18 Zhu ZW, Friess H, Wang L, et al. Enhanced glypican-3 expression differentiates the majority of hepatocellular carcinomas from benign hepatic disorders. Gut 2001;48:558-564. 
19 Ferrell LD, Crawford JM, Dhillon AP, et al. Proposal for standardized criteria for the diagnosis of benign, borderline and malignant hepatocellular lesions arising in chronic advanced liver disease. Am J Surg Pathol 1993;17:1113-1123.

20 Wanless IR, Callea F, Craig JR, et al. Terminology of nodular hepatocellular lesions. International Working Party. Hepatology 1995;22:983-993.

21 Hirohashi S, Ishak KG, Kojiro M, et al. Hepatocellular carcinoma. In: Hamilton SR, Aaltonen LA (eds). Pathology and genetics of tumors of the digestive system. IARC Press: Lyon, France, 2000, pp 159-172.

22 Kojiro M. Focus on dysplastic nodules and early hepatocellular carcinoma: an Eastern point of view. Liver Transpl 2004;10(Suppl 1):S3-S8.

23 Hytiroglou P. Morphological changes of early human hepatocarcinogenesis. Semin Liver Dis 2004;24:65-75.

24 Hsu HC, Cheng W, Lai PL. Cloning and expression of a developmentally regulated transcript MXR7 in hepatocellular carcinoma: biological significance and temporospatial distribution. Cancer Res 1997;57: 5179-5184.

25 Farooq M, Hwang SY, Park MK, et al. Blocking endogenous glypican-3 expression releases Hep 3B cells from G1 arrest. Mol Cells 2003;15:356-360.

26 Capurro MI, Xiang YY, Lobe C, et al. Glypican-3 promotes the growth of hepatocellular carcinoma by stimulating canonical Wnt signaling. Cancer Res 2005;65:6245-6254.

27 Kwack MH, Choi BY, Sung YK. Cellular changes resulting from forced expression of glypican-3 in hepatocellular carcinoma cells. Mol Cells 2006;21: 224-228.

28 Di Tommaso L, Franchi G, Park YN, et al. Diagnostic value of HSP70, glypican 3, and glutamine synthetase in hepatocellular nodules in cirrhosis. Hepatology 2007;45:725-734.

29 Ruffin IV MT. Fibrolamellar hepatoma. Am J Gastroenterol 1990;85:577-581.

30 Berman MA, Burnham JA, Sheahan DG. Fibrolamellar carcinoma of the liver: an immunohistochemical study of nineteen cases and a review of the literature. Hum Pathol 1988;19:784-794.

31 Matsuura S, Aishima S, Taguchi K, et al. 'Scirrhous' type hepatocellular carcinomas: a special reference to expression of cytokeratin 7 and hepatocyte paraffin 1 . Histopathology 2005;47:382-390.

32 Nakatsura T, Kageshita T, Ito S, et al. Identification of glypican-3 as a novel tumor marker for melanoma. Clin Cancer Res 2004;10:6612-6621.

33 Nakatsura T, Nishimura Y. Usefulness of the novel oncofetal antigen glypican-3 for diagnosis of hepatocellular carcinoma and melanoma. BioDrugs 2005;19: 71-77.

34 Stadlmann S, Gueth U, Baumhoer D, et al. Glypican-3 expression in primary and recurrent ovarian carcinomas. Int J Gynecol Pathol 2007;26: 341-344.

35 Abdul-Al HM, Makhlouf HR, Wang G, et al. Glypican-3 expression in benign liver tissue with active hepatitis C: implications for the diagnosis of hepatocellular carcinoma. Hum Pathol 2008;39:209-212. 